\title{
Coexistence of magnetism and superconductivity in $\mathrm{Eu}_{1.4} \mathrm{Ce}_{0.6} \mathrm{RuSr}_{2} \mathrm{Cu}_{2} \mathrm{O}_{10}$ : A muon spin rotation and magnetization study
}

\author{
A. Shengelaya, ${ }^{1}$ R. Khasanov, ${ }^{1,2}$ D. G. Eshchenko, ${ }^{1}$ I. Felner, ${ }^{3}$ U. Asaf, ${ }^{3}$ I. M. Savić, ${ }^{4}$ H. Keller, ${ }^{1}$ and K. A. Müller ${ }^{1}$ \\ ${ }^{1}$ Physik-Institut der Universität Zürich, CH-8057 Zürich, Switzerland \\ ${ }^{2}$ Paul Scherrer Institut, CH-5232 Villigen PSI, Switzerland \\ ${ }^{3}$ Racah Institute of Physics, The Hebrew University, Jerusalem 91904, Israel \\ ${ }^{4}$ Faculty of Physics, University of Belgrade, 11001 Belgrade, Serbia and Montenegro
}

(Received 28 March 2003; published 26 January 2004)

\begin{abstract}
We performed zero-field muon spin rotation (ZF- $\mu \mathrm{SR})$ and $\mathrm{dc}$ magnetization experiments in the ruthenatecuprate $\mathrm{Eu}_{1.4} \mathrm{Ce}_{0.6} \mathrm{RuSr}_{2} \mathrm{Cu}_{2} \mathrm{O}_{10}$. It was found that this compound becomes weakly ferromagnetic at $T_{m}$ $=77.6 \mathrm{~K}$ and superconducting below $T_{c}=40 \mathrm{~K}$. ZF- $\mu$ SR experiments indicate that the magnetic order accounts for most of the sample volume and magnetism is not significantly affected in the superconducting state. The present investigation provides evidence for the coexistence of magnetism and superconductivity in $\mathrm{Eu}_{1.4} \mathrm{Ce}_{0.6} \mathrm{RuSr}_{2} \mathrm{Cu}_{2} \mathrm{O}_{10}$.
\end{abstract}

DOI: 10.1103/PhysRevB.69.024517

PACS number(s): 74.72.Jt, 75.50.-y, 76.75.+i

\section{INTRODUCTION}

Recently, the hybrid ruthenocuprates $\mathrm{RuSr}_{2}\left(\mathrm{R}_{2-x} \mathrm{Ce}_{x}\right) \mathrm{Cu}_{2} \mathrm{O}_{10}(\mathrm{Ru}-1222)$ and $\mathrm{RuSr}_{2} R \mathrm{Cu}_{2} \mathrm{O}_{8}(\mathrm{Ru}-$ 1212) $(R=\mathrm{Sm}, \mathrm{Eu}$, and $\mathrm{Gd})$ have attracted much attention because of the coexistence of superconductivity and longrange magnetic order. ${ }^{1,2}$ These compounds have a layered structure with alternating $\mathrm{CuO}_{2}$ and $\mathrm{RuO}_{2}$ layers. It seems that superconductivity takes place in the $\mathrm{CuO}_{2}$ layers like in the high- $T_{c}$ cuprates, whereas magnetic order occurs in the $\mathrm{RuO}_{2}$ planes. Till now most of the reports have focused on the Ru-1212 phase, where the magnetic transition is observed at $T_{m}=133 \mathrm{~K}$ and superconductivity sets in at $T_{c}$ $=35 \mathrm{~K}^{2-5}$ These experiments provide the evidence for the coexistence of superconductivity and magnetism in Ru-1212 compound. However, many important questions such as the nature of superconductivity, details of the magnetic order, and the mechanism of coexistence of superconductivity and magnetism still remain a matter of discussion.

In this paper we focus our attention on a Ru-1222 phase $\mathrm{Eu}_{1.4} \mathrm{Ce}_{0.6} \mathrm{RuSr}_{2} \mathrm{Cu}_{2} \mathrm{O}_{10}$, which is actually the first ruthenocuprate where the coexistence of superconductivity and magnetism was found. ${ }^{1}$ Both Ru-1212 and Ru-1222 contain similar $\mathrm{CuO}_{2}$ and $\mathrm{RuO}_{2}$ planes. However, in $\mathrm{Ru}-1222$ the $R$ layer between $\mathrm{CuO}_{2}$ planes is replaced by a fluoritetype $\left(R_{2-x} \mathrm{Ce}_{x} \mathrm{O}_{2} ; R=\mathrm{Gd}, \mathrm{Eu}\right)$ layer. This allows to vary the charge-carrier concentration in $\mathrm{Ru}-1222$, in contrast to Ru-1212 which is a stoichiometric compound. Recently, a jump in the specific heat at $T_{c}$ was observed in $\mathrm{Gd}_{1.4} \mathrm{Ce}_{0.6} \mathrm{RuSr}_{2} \mathrm{Cu}_{2} \mathrm{O}_{10}$, confirming bulk superconductivity. ${ }^{6,7}$ However, no microscopic measurement of the magnetic state has been reported up to now and it remains to be demonstrated that magnetic order also has bulk character in order to infer the coexistence of magnetism and superconductivity in Ru-1222. In addition, the magnetic properties of Ru-1222 are more complicated than that of Ru-1212. Several magnetic transitions were found in magnetization measurements. ${ }^{1}$ From the magnetization data alone it is practically impossible to determine the nature of these magnetic transitions. To answer these questions we studied $\mathrm{Eu}_{1.4} \mathrm{Ce}_{0.6} \mathrm{RuSr}_{2} \mathrm{Cu}_{2} \mathrm{O}_{10}$ using zero-field muon spin rotation (ZF- $\mu \mathrm{SR}$ ). The $\mu \mathrm{SR}$ technique is especially suitable for such a study since the positive muon is an extremely sensitive local probe to detect internal magnetic fields and their distribution. In addition, as a real-space probe, $\mu \mathrm{SR}$ can determine the ordered volume fraction.

This paper is organized as follows. Sample preparation and principles of the $\mu \mathrm{SR}$ technique are briefly described in Sec. II. In Sec. III we present dc magnetization, resistivity, and $\mathrm{ZF}-\mu \mathrm{SR}$ data and discuss the magnetic properties of $\mathrm{Eu}_{1.4} \mathrm{Ce}_{0.6} \mathrm{RuSr}_{2} \mathrm{Cu}_{2} \mathrm{O}_{10}$ based on the obtained data. In Sec. IV we summarize most important results and discuss a possible scenario for the coexistence of the magnetism and superconductivity in ruthenate-cuprates.

\section{EXPERIMENTAL DETAILS}

Ceramic samples with nominal composition $\mathrm{Eu}_{1.4} \mathrm{Ce}_{0.6} \mathrm{RuSr}_{2} \mathrm{Cu}_{2} \mathrm{O}_{10}$ were prepared by a solid-state reaction technique. Prescribed amounts of $\mathrm{Eu}_{2} \mathrm{O}_{3}, \mathrm{CeO}_{2}$, $\mathrm{SrCO}_{3}, \mathrm{Ru}$, and $\mathrm{CuO}$ were mixed and pressed into pellets and preheated at $1000^{\circ} \mathrm{C}$ for about 1 day in the presence of flowing oxygen at atmospheric pressure. The product was cooled, reground, and sintered at $1050{ }^{\circ} \mathrm{C}$ for $72 \mathrm{~h}$ in a slightly pressurized oxygen atmosphere $(\sim 1.1 \mathrm{~atm})$ and then cooled in a furnace to ambient temperature. Powder x-ray diffraction measurements confirmed the purity of the sample (97\%) and provided the lattice parameters, $a=3.846(1) \AA$ and $c=28.72(1) \AA$.

The dc magnetization data were obtained using a Quantum Design superconducting quantum interference device magnetometer. Resistivity measurements were performed with a standard four-probe dc technique on bar-shaped pieces cut out from the pellets. The contacts were made with silver paint. The $\mu \mathrm{SR}$ experiments were performed on the beam line $\pi M 3$ at the Paul Scherrer Institute (Switzerland) using positive low-momentum $(29 \mathrm{MeV} / c)$ "surface muons." The essentially $100 \%$ spin-polarized muons are implanted into the bulk of the sample where they thermalize very rapidly 


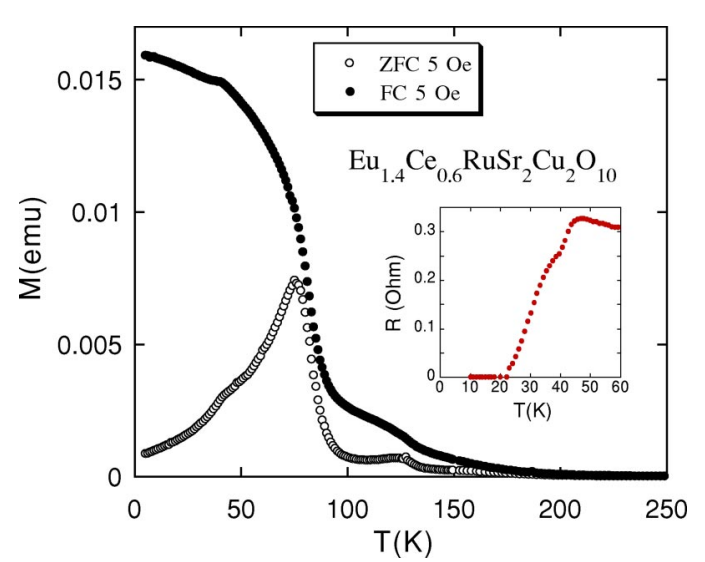

FIG. 1. Temperature dependence of the zero-field-cooled (ZFC) and field-cooled (FC) dc magnetization of $\mathrm{Eu}_{1.4} \mathrm{Ce}_{0.6} \mathrm{RuSr}_{2} \mathrm{Cu}_{2} \mathrm{O}_{10}$. Inset: resistivity data showing the superconducting transition.

$\left(\sim 10^{-12} \mathrm{~s}\right)$ without any significant loss in their initial spin polarization. The muon decays with a mean lifetime of $\tau_{\mu^{+}}$ $=2.2 \mu \mathrm{s}$ into a positron and two undetected neutrinos. Due to parity violation the decay positron is emitted preferentially along the muon spin direction, and by collecting several hundreds of thousands positrons the muon spin depolarization function is reconstructed which, in turn, reflects the spatial and temporal distribution of magnetic fields at the muon site. Because of its positive charge, the muon localizes at an interstitial lattice site and in the perovskite compounds forms a muoxyl bond with oxygen atoms. ${ }^{8}$ The whole ensemble of muons is randomly distributed throughout a layer of 100-200 $\mu \mathrm{m}$ thickness and therefore probes a representative part of the sample volume.

In ZF- $\mu$ SR experiments the time evolution of the muon spin polarization is monitored with no external field applied, reflecting the interaction of the implanted muon spin with the local magnetic field at the muon site $\mathrm{B}_{\mu}$ in the bulk of the sample (the earth's magnetic field was compensated to better than $10 \mu \mathrm{T})$. Each muon spin precesses in its local magnetic field $\mathrm{B}_{\mu}$ with a precession frequency of $\nu_{\mu}=\left(\gamma_{\mu} / 2 \pi\right) B_{\mu}$, where $\gamma_{\mu} / 2 \pi=135.5 \mathrm{MHz} / \mathrm{T}$ is the gyromagnetic ratio of the positive muon. For a detailed description of the basic concepts of $\mu \mathrm{SR}$ and its application to study the magnetic properties of solids we refer to the literature. ${ }^{9}$

\section{RESULTS AND DISCUSSION}

\section{A. dc magnetization}

Figure 1 shows the temperature dependence of the zerofield cooled (ZFC) and the field-cooled (FC) magnetization for the $\mathrm{Eu}_{1.4} \mathrm{Ce}_{0.6} \mathrm{RuSr}_{2} \mathrm{Cu}_{2} \mathrm{O}_{10}$ sample. There is a strong increase of magnetization below $T_{m} \sim 90 \mathrm{~K}$ with a large difference between ZFC and FC measurements, in agreement with a previous report. ${ }^{1} \mathrm{~A}$ similar magnetic transition was also observed in Ru-1212 compound, ${ }^{10}$ which is consistent with canted antiferromagnetic order with a small ferromagnetic moment in accordance with a neutron-diffraction study. ${ }^{11} \mathrm{Be}-$ low $T_{c} \sim 40 \mathrm{~K}$ the ZFC magnetization shows an additional decrease. This corresponds to the superconductivity onset

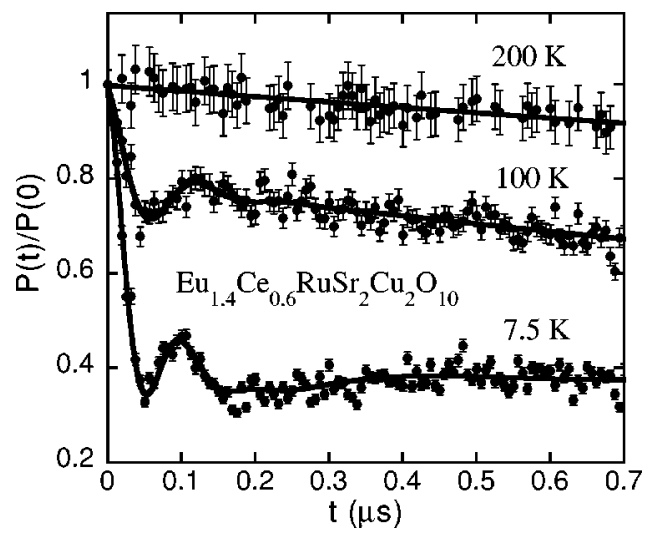

FIG. 2. Zero-field (ZF) $\mu$ SR spectra of $\mathrm{Eu}_{1.4} \mathrm{Ce}_{0.6} \mathrm{RuSr}_{2} \mathrm{Cu}_{2} \mathrm{O}_{10}$ for three different temperatures. Solid lines represent fits using Eq. (1).

temperature determined from the resistivity measurements (see inset of Fig. 1). The superconducting transition is also reflected in FC magnetization as a small kink near $40 \mathrm{~K}$, similar to a superconducting Meissner transition with a small volume fraction. Generally, this anomaly was found to be sample dependent and is strongly suppressed by an external magnetic field. A similar decrease of the FC magnetization in the superconducting state was previously reported for Ru-1212, ${ }^{12}$ but not for Ru-1222. ${ }^{1}$ The small volume of the Meissner fraction of the Ru-1222 samples, similar to $\mathrm{Ru}-$ 1212 , may raise the question concerning the impurity nature of superconductivity in these compounds. ${ }^{13}$ We note, however, that strong evidence for bulk superconductivity state in Ru-1222 comes from recent specific-heat measurements, where a sizable jump in the specific-heat coefficient $\gamma$ was observed at $T_{c}$, comparable to underdoped high- $T_{c}$ superconductors. ${ }^{6,7}$ Recent dc magnetization measurements on Ru-1212 samples provided evidence that a bulk Meissner state develops at low magnetic fields $H<1$ Oe and temperatures much below $T_{c}$, which is sample dependent. ${ }^{12}$ This peculiar behavior could be attributed to the spontaneous vortex phase. We will discuss this phase in more detail later.

In addition to a large increase of the magnetization below $90 \mathrm{~K}$, there is a small peak in the ZFC data near $130 \mathrm{~K}$ (see Fig. 1). Moreover, there is a difference between $\mathrm{ZFC}$ and FC magnetization curves which extends up to $T_{i r r} \sim 200 \mathrm{~K}$. A similar behavior of magnetization was reported by other groups, and it seems to be an intrinsic property of Ru-1222. ${ }^{14}$ From the magnetization data alone it is very difficult to identify the nature of these magnetic anomalies. Therefore we performed ZF- $\mu$ SR experiments to study the complex magnetic properties of Ru-1222 on a microscopic level.

\section{B. Zero-field muon spin rotation}

Figure 2 shows a representative ZF- $\mu$ SR spectra for the $\mathrm{Eu}_{1.4} \mathrm{Ce}_{0.6} \mathrm{RuSr}_{2} \mathrm{Cu}_{2} \mathrm{O}_{10}$ sample at different temperatures. The clear oscillation observed in the time spectra at low temperatures implies that the muons sense a well-defined internal magnetic field. The $\mu \mathrm{SR}$ spectra were analyzed using the standard function ${ }^{9}$ 


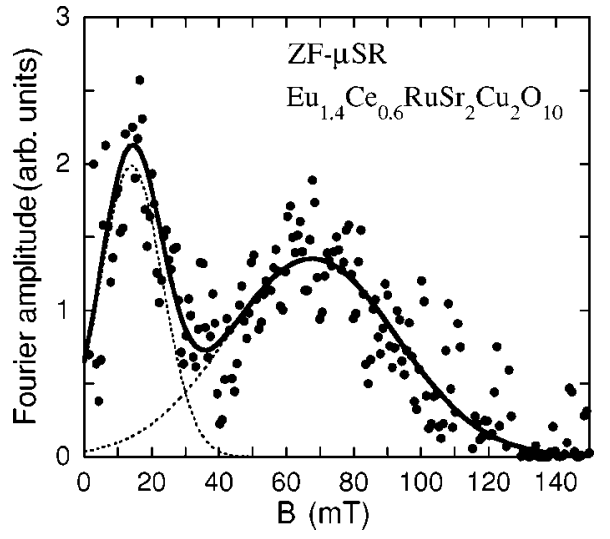

FIG. 3. Fourier-transformed $\mathrm{ZF}-\mu \mathrm{SR}$ spectrum of $\mathrm{Eu}_{1.4} \mathrm{Ce}_{0.6} \mathrm{RuSr}_{2} \mathrm{Cu}_{2} \mathrm{O}_{10}$ at $T=5 \mathrm{~K}$. A double peak in the internal magnetic-field distribution is clearly seen. The solid line is a fit with a sum of two Gaussians represented by dashed lines.

$$
A(t)=A_{\|} \exp (-\lambda t)+A_{\perp} \exp \left(-\Delta^{2} t^{2} / 2\right) \cos \left(\gamma_{\mu} B_{\mu} t+\Phi\right),
$$

where $\gamma_{\mu} / 2 \pi=135.538 \mathrm{MHz} \mathrm{s}^{-1} \mathrm{~T}^{-1}$ is the gyromagnetic ratio of the positive muon and $\mathrm{B}_{\mu}$ the average static internal magnetic field at the muon site. In the absence of an external magnetic field, $\mathrm{B}_{\mu}$ is determined by the local magnetic order parameter, i.e., the staggered magnetization. The two components are due to muons with $\mathrm{B}_{\mu}$ parallel or transverse to the muon spin polarization. The corresponding relaxation rates are $\lambda$ (longitudinal) and $\Delta$ (transverse).

We observed that a fit to Eq. (1) below $90 \mathrm{~K}$ with a single $\mathrm{B}_{\mu}$ resulted in a very poor agreement with the spectra. In case of an incommensurate magnetic order the cosine function in Eq. (1) can change to a Bessel function. ${ }^{15}$ However, the Bessel function did not improve the fit either. We found that two different local magnetic fields $\mathrm{B}_{\mu_{1}}$ and $\mathrm{B}_{\mu_{2}}$ satisfactorily fit the spectra below $90 \mathrm{~K}$. This was further confirmed by a Fourier transformation of the time spectra, where two peaks in the magnetic-field distribution were observed (see Fig. 3). It is seen from Fig. 2 that the oscillations are present also above $90 \mathrm{~K}$ but with a much smaller amplitude. This indicates that some magnetic order still exists above this temperature.

In Fig. 4(a) the internal magnetic fields $\mathrm{B}_{\mu}$ are shown as functions of temperature. The solid lines are fits to the phenomenological power-law temperature dependence $B_{\mu}(T)$ $=B_{\mu}(0)\left(1-T / T_{m}\right)^{n}$. The obtained parameters are summarized in Table I. One can see that there are two oscillating signals which correspond to the large magnetic fields at the muon sites $\mathrm{B}_{\mu}^{H F 1}(0)=74(10) \mathrm{mT}$ and $\mathrm{B}_{\mu}^{H F 2}(0)=59(1) \mathrm{mT}$ with transition temperatures $T_{m 1} \simeq 200 \mathrm{~K}$ and $T_{m 2} \simeq 95 \mathrm{~K}$, respectively. We call them high-field (HF1 and HF2) signals. In addition, there is one oscillating signal with much lower field $\mathrm{B}_{\mu}^{L F}(0)=12.5(1) \mathrm{mT}$ below $T_{m 3}=77.6 \mathrm{~K}$, which we call low-field (LF) signal.

The present results provide evidence of a magnetically ordered state in $\mathrm{Eu}_{1.4} \mathrm{Ce}_{0.6} \mathrm{RuSr}_{2} \mathrm{Cu}_{2} \mathrm{O}_{10}$. Moreover, it is clear that the magnetic order persists almost unchanged in the superconducting state since there is no anomaly of the
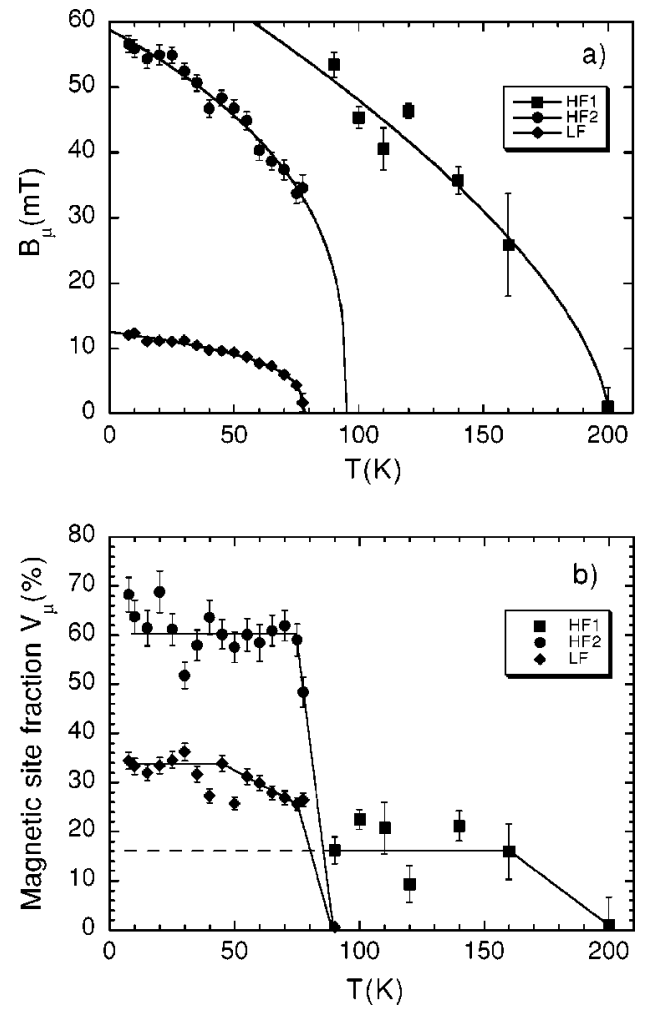

FIG. 4. (a) Temperature dependence of the different internal magnetic fields $B_{\mu}(\mathrm{HF} 1, \mathrm{HF} 2, \mathrm{LF})$ in $\mathrm{Eu}_{1.4} \mathrm{Ce}_{0.6} \mathrm{RuSr}_{2} \mathrm{Cu}_{2} \mathrm{O}_{10}$. The solid lines represent fitted curves to the power law $B_{\mu}(T)=B_{\mu}(0)$ $\times\left(1-T / T_{m}\right)^{n}$. (b) Temperature dependence of the volume fraction $V_{\mu}$ of the muon sites that experience an internal magnetic field. The lines are guides for the eye.

$\mathrm{ZF}-\mu \mathrm{SR}$ signal below the superconducting transition temperature $T_{c}=40 \mathrm{~K}$. Now the important question arises whether this magnetic order has a bulk character or occurs only in a small part of the sample. It is possible to extract the volume fraction of the magnetically ordered phase from the amplitude of the oscillating component of the ZF- $\mu \mathrm{SR}$ spectra. In a magnetically ordered polycrystalline sample with randomly oriented grains in zero external field, the internal magnetic field is parallel (perpendicular) to the muon spin direction with a probability $1 / 3(2 / 3)$. For a homogeneous magnetically ordered sample, one therefore expects that $2 / 3$ of the amplitude of the $\mathrm{ZF}-\mu \mathrm{SR}$ signal exhibits an oscillatory behavior (transverse component) $A_{\perp} /\left(A_{\perp}+A_{\|}\right)=2 / 3$, while $1 / 3$ of the signal is nonoscillating (longitudinal component) and only slowly damped.

On the other hand, if the sample contains nonmagnetic regions, the amplitude of the oscillating signal will be ac-

TABLE I. $\mathrm{T}_{m}, B_{\mu}(0)$, and $n$ derived by fitting the measured values of $B_{\mu}(T)$ to the power law $B_{\mu}(T)=B_{\mu}(0)\left(1-T / T_{m}\right)^{n}$ (see text).

\begin{tabular}{cccc}
\hline \hline$\mu$ SR signal & $T_{m}(\mathrm{~K})$ & $B_{\mu}(0)(\mathrm{mT})$ & $n$ \\
\hline HF1 & $200(10)$ & $74(10)$ & $0.6(1)$ \\
HF2 & $95(7)$ & $59(1)$ & $0.34(6)$ \\
LF & $77.6(1)$ & $12.5(1)$ & $0.31(1)$ \\
\hline \hline
\end{tabular}


cordingly reduced. For a polycrystalline sample where only a $V_{o r}$ portion of the total volume $V_{\text {to }}$ orders, $A_{\perp} /\left(A_{\perp}+A_{\|}\right)$ $=\frac{2}{3} V_{o r} / V_{t o}<\frac{2}{3}$. We determined the amplitude of the total initial muon spin polarization $P(t=0)$ from transverse-field measurements at $T=300 \mathrm{~K}$ where the samples were nonmagnetic. The volume fraction $V_{\mu}$ of muon sites that experience the internal magnetic field was determined as $V_{\mu}$ $=V_{o r} / V_{t o}$. Figure 4(b) shows the temperature dependence of the volume fraction $V_{\mu}$ of muon sites that experience the internal magnetic field. One can see that the oscillating signal HF1 appears below $200 \mathrm{~K}$ and its asymmetry is practically constant down to $90 \mathrm{~K}$. From the amplitude of this oscillatory component we deduce that only about $15(5) \%$ of the sample is magnetically ordered in the temperature range 90-200 K. Below $90 \mathrm{~K}$ two new oscillatory signals HF2 and LF with much higher amplitudes appear in the $\mu$ SR spectra. From the total amplitude of the oscillating components we deduce that about $95(5) \%$ of the sample volume is magnetically ordered below $90 \mathrm{~K}$. It means that practically all sample volume is magnetically ordered above $T_{c}=40 \mathrm{~K}$. Moreover, Fig. 4 shows that the magnetic order persists almost unchanged in the superconducting state. One can ask what happens to the HF1 signal below $90 \mathrm{~K}$. The amplitude of the HF1 signal is only $15(5) \%$ which is in the limit of the $\mu \mathrm{SR}$ sensitivity. It becomes very difficult to resolve this small component below $90 \mathrm{~K}$ where much stronger oscillating signals appear in the $\mu \mathrm{SR}$ spectra. The situation is further complicated by the fact that the HF1 and HF2 signals have close frequencies. Therefore one cannot follow the HF1 signal below $90 \mathrm{~K}$.

The presence of the two oscillating components (HF2 and LF) in EuRu-1222 at low temperatures is in contrast to $\mathrm{Ru}-$ 1212 where only a single muon spin precession signal was observed. ${ }^{2}$ Our $\mu$ SR signals correspond to two very different internal magnetic fields $B_{\mu}(0)=59(1)$ and $12.5(1) \mathrm{mT}$, respectively. The ratio of the two muon signal amplitudes $A_{H F 2} / A_{L F} \approx 2$ and is practically temperature independent. One should note that $B_{\mu}^{H F 2}(0)=59(1) \mathrm{mT}$ is close to $B_{\mu}(0)=72(1) \mathrm{mT}$ observed in Ru-1212 sample by $\mu \mathrm{SR},{ }^{2}$ but $B_{\mu}^{L F}(0)$ is much smaller. The two oscillating signals are most probably due to two different muon stopping sites in the Ru-1222 structure. Both Ru-1212 and Ru-1222 contain similar $\mathrm{CuO}_{2}$ and $\mathrm{RuO}_{2}$ planes. However, $\mathrm{Ru}-1222$ contains in addition a fluorite type $\left(R_{2-x} \mathrm{Ce}_{x} \mathrm{O}_{2} ; R=\mathrm{Gd}, \mathrm{Eu}\right)$ layer between the $\mathrm{CuO}_{2}$ planes. It is possible that the second muon stopping site is close to the $\mathrm{R}_{2-x} \mathrm{Ce}_{x} \mathrm{O}_{2}$ layer. At this site muons will be far away from magnetically ordered $\mathrm{RuO}_{2}$ planes, feeling a much reduced internal magnetic field in agreement with the experiment. In the case of two muon stopping sites we would expect the two oscillating signals having the same transition temperature. The fit showed that the HF2 signal has a transition temperature of $95 \mathrm{~K}$ which is higher than $78 \mathrm{~K}$ for the LF signal. However, one should note that the transition temperature of $95 \mathrm{~K}$ is obtained from the fit without having experimental points close to this temperature. The reason for this is that below $90 \mathrm{~K}$ the HF1 and HF2 signals overlap, and it is very difficult to separate them unambiguously especially because their oscillation frequen- cies are close to each other as we already mentioned before. We think that the transition temperature of $95 \mathrm{~K}$ for the HF2 signal is artificially enhanced due to the interference with the HF1 signal and the intrinsic magnetic transition is lower and close to $77.6 \mathrm{~K}$ obtained from the temperature dependence of the LF signal.

We ascribed two oscillating components (HF2 and LF) to two muon stopping sites within the same magnetic phase. However, based on the obtained $\mu$ SR data we cannot exclude that the LF and HF2 signals extrapolate to different transition temperatures. Especially, it is open whether the HF2 signal does not indicate a first-order transition at $78 \mathrm{~K}$. As this may be the LF and HF2 signals can belong to two different magnetic regions. Such a scenario was recently proposed in $\mathrm{Ru}-1222$ based on the magnetization measurements. ${ }^{16}$ It is clear that Ru-1222 shows more complex magnetic behavior than Ru-1212, which requires further studies in particular with neutron diffraction.

\section{Magnetic transitions in $\mathrm{Eu}_{1.4} \mathrm{Ce}_{0.6} \mathrm{RuSr}_{2} \mathrm{Cu}_{2} \mathrm{O}_{10}$ as revealed by $\mu \mathrm{SR}$}

As one can see from Fig. 1, the magnetization behavior of $\mathrm{Eu}_{1.4} \mathrm{Ce}_{0.6} \mathrm{RuSr}_{2} \mathrm{Cu}_{2} \mathrm{O}_{10}$ can be divided basically into two regions, going from high to low temperatures. First, below $T_{i r r} \simeq 200 \mathrm{~K}$ the irreversibility appears between the ZFC and FC data. Below $T_{m} \simeq 90 \mathrm{~K}$ there is an additional sharp increase of the magnetization and very strong irreversibility between the ZFC and FC branches. This behavior is typical for Ru-1222 and it was proposed that at $T_{i r}$ the Ru spins order antiferromagnetically and below $T_{m}$ weak ferromagnetism arises due to the canting of the Ru moments. ${ }^{1}$ This scenario was supported by Mössbauer spectroscopy measurements which revealed magnetic hyperfine splitting in $\mathrm{Ru}$ 1222 samples below $T_{i r r}$, indicating magnetic order. ${ }^{1}$

Our $\mu \mathrm{SR}$ results indeed confirm the presence of magnetic order below $200 \mathrm{~K}$. However, they show that magnetism takes place only in a small portion $(\sim 15 \%)$ of the sample. Therefore there is no bulk magnetic order in Ru-1222 in the temperature range $T_{m}<T<T_{i r r}$. It is important to point out that this magnetic order is not due to impurities. X-ray diffraction showed that our samples are single phase and any impurity content must be below 5\%. This excludes impurity phases as a reason of magnetism below $T_{i r r}$. Recently, formation of magnetic clusters (nanodomains) in Ru-1222 below $T_{i r r}$ was proposed from the magnetization measurements. ${ }^{16}$ The partial magnetic order below $200 \mathrm{~K}$ observed in our $\mu \mathrm{SR}$ experiments is rather consistent with the existence of such magnetic clusters in the temperature range $T_{m}<T$ $<T_{i r r}$. However, the amplitude of the oscillating muon signal is fairly temperature independent in this temperature region [see Fig. 4(b)]. This shows that the magnetic nanodomains, if existing, do not behave as typical superparamagnetic particles. Note that for the superparamagnetic clusters the magnetic volume fraction is temperature dependent.

It is instructive to compare the magnetic transition temperatures obtained by ZF- $\mu \mathrm{SR}$ with the magnetization data presented in Fig. 1. It is seen that the magnetic transition 
observed with $\mu$ SR below $\sim 200 \mathrm{~K}$ corresponds to $T_{i r r}$ in the magnetization data where small irreversibility between ZFC and FC starts to appear. Below $\sim 90 \mathrm{~K}$ the magnetization strongly increases and a very large irreversibility between the ZFC and FC branches is observed. This corresponds to the sharp increase of the magnetic volume fraction observed by $\mu$ SR. Finally, a well resolved internal magnetic field appears at $T_{m}=77.6 \mathrm{~K}$, which approximately corresponds to the temperature $T_{p}$ where the ZFC magnetization has a peak. Below $T_{p}$ the ZFC magnetization decreases. We note that the small peak observed in the ZFC magnetization near $\sim 130 \mathrm{~K}$ (see Fig. 1) is not reflected in the $\mu$ SR data, suggesting that the magnetism related with this anomaly does not have a bulk character.

\section{SUMMARY AND CONCLUSIONS}

In summary, we performed resistivity, dc magnetization, and ZF- $\mu$ SR experiments in the $\mathrm{Eu}_{1.4} \mathrm{Ce}_{0.6} \mathrm{RuSr}_{2} \mathrm{Cu}_{2} \mathrm{O}_{10}$ ruthenocuprate. ZF- $\mu$ SR showed that $15(5) 5 \%$ of the sample volume orders magnetically below $\sim 200 \mathrm{~K}$. This corresponds to the onset of irreversibility in the magnetization data at $T_{i r r}$ where a difference between ZFC and FC branches appears. The observed partial magnetic order below $T_{i r r}$ is consistent with the existence of small magnetic regions in Ru-1222 which was inferred from the recent detailed magnetization measurements. ${ }^{16}$ With decreasing temperature there is a bulk magnetic transition at $T_{m}=77.6 \mathrm{~K}$, and the magnetic phase accounts for the whole sample volume. Below $T_{c}=40 \mathrm{~K}$ the sample becomes superconducting and magnetic order persists unchanged in the superconducting state.

Taking into account the specific-heat measurements of Ru-1222, demonstrating bulk superconductivity, ${ }^{6,7}$ and the present results, we conclude that in Ru-1222 magnetism coexists with bulk superconductivity. Such a coexistence implies that the magnetism is related to the $\mathrm{RuO}_{2}$ layers which are very weakly coupled to the superconducting $\mathrm{CuO}_{2}$ planes. Generally, one may conclude that despite some structural differences between the Ru-1222 and Ru-1212 ruthenate-cuprates, they have similar physical properties. The most remarkable one is the microscopic coexistence of superconductivity and weak ferromagnetism. Such a coexistence is very surprising in view of the antagonistic nature of superconductivity (SC) and ferromagnetism (FM). Many years ago it was proposed theoretically that exotic phases with coexistence of superconductivity and magnetism may occur in FM superconductors. ${ }^{17,18}$ In particular, it is possible that the spontaneous FM moments give rise to the so-called spontaneous vortex phase (SVP), so that the ferromagnetism coexists with the superconductivity. This can happen because the self-energy of a vortex becomes negative under an appropriate condition in the FM superconductor, and thus the vortices would be induced without applying an external magnetic field. A necessary condition for the SVP state was given by $H_{c 1}^{0}<M_{F M}<H_{c 2}^{0}$, where $H_{c 1}^{0}$ and $H_{c 2}^{0}$ are the first and second critical fields for a nonmagnetic superconductor and $M_{F M}$ is the average spontaneous FM magnetization. One can see that $M_{F M}$ plays a role of the external magnetic field. In the SVP the sample is in the mixed state, even at zero applied magnetic field, and the Meissner state is absent. The Meissner state is possible only below the temperature where $M_{F M}$ becomes smaller than $H_{c 1}^{0}$. Such a behavior would be consistent with the magnetization measurements in Ru-1212 and Ru-1222 compounds. ${ }^{12,19}$

One can see that SVP explains the microscopic coexistence of SC and FM in ruthenate-cuprates as well as their peculiar SC properties. Now the question arises whether the SVP indeed exists in these compounds. Estimations showed that in ruthenate-cuprates $H_{c 1}^{0}$ and $M_{F M}$ are comparable, so that the formation of the SVP state is possible. ${ }^{19}$ Recently, using magneto-optical imaging it was demonstrated that in Ru-1222 magnetic flux is present below $T_{c}$ in zero applied magnetic field, ${ }^{20}$ but disappears above $T_{c}$. If confirmed, this would be the first direct observation of SVP. Up to now nearly all studies have been performed on polycrystalline ruthenate-cuprates. The availability of single crystalline and epitaxial thin-film samples would be important to further explore the peculiar superconducting and magnetic properties of these fascinating materials. In particular, it would be very interesting to perform small-angle neutron-scattering experiments to study SVP in the ruthenate-cuprates.

\section{ACKNOWLEDGMENTS}

This work was supported by the Swiss National Science Foundation, Israel Science Foundation, and Klachky Foundation for Superconductivity.
${ }^{1}$ I. Felner, U. Asaf, Y. Levi, and O. Millo, Phys. Rev. B 55, R3374 (1997).

${ }^{2}$ C. Bernhard, J.L. Tallon, Ch. Niedermayer, Th. Blasius, A. Golnik, E. Brücher, R.K. Kremer, D.R. Noakes, C.E. Stronach, and E.J. Ansaldo, Phys. Rev. B 59, 14099 (1999).

${ }^{3}$ A. Fainstein, E. Winkler, A. Butera, and J. Tallon, Phys. Rev. B 60, R12 597 (1999).

${ }^{4}$ O. Chmaissem, J.D. Jorgensen, H. Shaked, P. Dollar, and J.L. Tallon, Phys. Rev. B 61, 6401 (2000).

${ }^{5}$ J.L. Tallon, J.W. Loram, G.V.M. Williams, and C. Bernhard, Phys. Rev. B 61, R6471 (2000).
${ }^{6}$ X.H. Chen, Z. Sun, K.Q. Wang, S.Y. Li, Y.M. Xiong, M. Yu, and L.Z. Cao, Phys. Rev. B 63, 064506 (2001).

${ }^{7}$ X.H. Chen, Z. Sun, K.Q. Wang, Y.M. Xiong, H.S. Yang, H.H. Wen, Y.M. Ni, and Z.X. Zhao, J. Phys.: Condens. Matter 12, 10561 (2000).

${ }^{8}$ M. Weber, P. Birrer, F.N. Gygax, B. Hitti, E. Lippelt, H. Maletta, and A. Schenck, Hyperfine Interact. 63, 207 (1990); N. Nishida and H. Miyatake, ibid. 63, 183 (1990).

${ }^{9}$ See, e.g., P. Dalmas de Reotier and A. Yaouanc, J. Phys.: Condens. Matter 9, 9113 (1997), and references therein.

${ }^{10}$ G.V.M. Williams and S. Krämer, Phys. Rev. B 62, 4132 (2000). 
${ }^{11}$ J.W. Lynn, B. Keimer, C. Ulrich, C. Bernhard, and J.L. Tallon, Phys. Rev. B 61, R14 964 (2000).

${ }^{12}$ C. Bernhard, J.L. Tallon, E. Brücher, and R.K. Kremer, Phys. Rev. B 61, 14960 (2000).

${ }^{13}$ C.W. Chu, Y.Y. Xue, S. Tsui, J. Cmaidalka, A.K. Heilman, B. Lorenz, and R.L. Meng, Physica C 335, 231 (2000).

${ }^{14}$ B.D. Hennings, K.D.D. Rathnayaka, D.G. Naugle, and I. Felner, Physica C 370, 253 (2002); M.T. Escote, V.A. Meza, R.F. Jardim, L. Ben-Dor, M.S. Torikachvili, and A.H. Lacerda, Phys. Rev. B 66, 144503 (2002).

${ }^{15}$ A. Amato, R. Feyerherm, F.N. Gygax, A. Schenck, H.V. Löhney- sen, and H.G. Schlager, Phys. Rev. B 52, 54 (1995).

${ }^{16}$ Y.Y. Xue, D.H. Cao, B. Lorenz, and C.W. Chu, Phys. Rev. B 65 , 020511(R) (2001).

${ }^{17}$ M. Tachiki, H. Matsumoto, T. Koyama, and H. Umezawa, Solid State Commun. 34, 19 (1980).

${ }^{18}$ H.S. Greenside, E.I. Blount, and C.M. Varma, Phys. Rev. Lett. 46, 49 (1981).

${ }^{19}$ E.B. Sonin and I. Felner, Phys. Rev. B 57, R14 000 (1998).

${ }^{20}$ I. Felner, E.B. Sonin, T. Machi, and N. Koshizuka, Physica C 341-348, 715 (2000). 